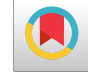

\title{
Novel Approaches to Immunotherapy in Triple Negative Breast Cancer
}

\author{
Sanaz Tabarestani (ii) ${ }^{1}$, Mohammad Esmaeil- Akbari (ii) ${ }^{1}$ and Saeed Namaki (iD ${ }^{2,{ }^{*}}$ \\ ${ }^{1}$ Cancer Research Center, Shahid Beheshti University of Medical Sciences, Tehran, Iran \\ ${ }^{2}$ Department of Medical Immunology, School of Medicine, Shahid Beheshti University of Medical Sciences, Tehran, Iran \\ "Corresponding author: Department of Medical Immunology, School of Medicine, Shahid Beheshti University of Medical Sciences, Tehran, Iran. Email: namaki@yahoo.com
}

Received 2018 December 03; Revised 2018 December 08; Accepted 2018 December 08.

\begin{abstract}
Context: Triple negative breast cancers (TNBC) constitute about 15\% of breast neoplasms. In contrast to estrogen receptor (ER) or human epidermal growth factor receptor (HER2) positive breast cancers, which respond to hormonal therapy (such as tamoxifen) or anti-HER2 therapy (such as trastuzumab), respectively, the main standard therapy in either early or late stage TNBC is chemotherapy. Therefore, it is necessary to find new treatment modalities for TNBC patients. We searched the literature to find published studies on immunotherapy in triple negative breast cancer and the putative biomarkers of response to these treatments.

Evidence Acquisition: We searched PubMed, Scopus, and Web of Science Core Collection with these keywords: "Triple negative breast cancer, Immunotherapy, Resistance, Response, Programmed cell death 1 receptor, CTLA-4, Tumor mutation burden, and Immune signature".

Results: TNBC is considered a heterogeneous neoplasm with regard to molecular aberrations. Analysis of genomic expression profile of TNBC has delineated 4 subtypes. TNBC tumors show high genetic instability. Tumor infiltrating lymphocytes (TILs) are detected more in TNBCs, compared to other breast cancer types. It has been shown that the amount of CD8 positive T cells in TNBCs is an independent predictor of overall survival. Up to now, two immunotherapy strategies have been used in clinical trials of TNBC, including immune checkpoint blockers and therapeutic cancer vaccines. Tumor programmed cell death ligand 1(PDL1) expression is the most widely used immunotherapy biomarker. Tumor mutation burden (TMB) can be a promising biomarker of response to immunotherapy. The more somatic mutations a cancer cell has, the more neoantigens it probably produces. Analysis of TMB can give an estimate of tumor mutation load. Increased somatic mutation load has also been observed in tumors with impaired mismatch repair (MMR).

Conclusions: As TNBC is regarded a heterogeneous disease, the discovery of biomarkers of response to immunotherapy will increase the likelihood of response to these therapies. Further in-depth investigations are needed to find novel biomarkers of response to these immunotherapies for the better management of patients with TNBC.
\end{abstract}

Keywords: Immunotherapy, Triple Negative Breast Cancer, Immune Signature, Biomarker

\section{Context}

Breast cancer is the most prevalent cancer among women in the world (1). Triple negative breast cancers (TNBC), which constitute about $15 \%$ of breast neoplasms, do not express estrogen receptor (ER), progesterone receptor (PR), and human epidermal growth factor receptor (HER2) (2, 3). TNBC is considered the most fatal type of breast cancer and patients diagnosed with TNBC have worse clinical outcome in comparison to other types (2, 4$6)$. In contrast to ER or HER2 positive breast cancers which respond to hormonal therapy (such as tamoxifen) or antiHER2 therapy (such as trastuzumab), respectively, the main standard therapy in either early or late stage TNBC is chemotherapy $(7,8)$. In addition, response to chemotherapy is usually short-lived and treatment of these patients is considered challenging (9). Therefore, it is necessary to find new treatment modalities for TNBC patients.

In recent years, cancer immunotherapy has developed considerably, including the use of checkpoint blockers such as antibodies against programmed cell death protein 1 (PD1) or cytotoxic T lymphocyte associated protein 4 (CTLA4), therapeutic cancer vaccines and adoptive cell transfer using chimeric antigen receptor (CAR) T-cell therapy (10). Multiple trials are analyzing the efficacy of immunotherapy in breast cancer. We searched the literature to find relevant published studies on triple negative breast cancer and the putative biomarkers of response to these treatments.

\section{Evidence Acquisition}

We searched PubMed, Scopus, and Web of Science Core Collection with these keywords: "Triple negative 
breast cancer, Immunotherapy, Resistance, Response, Programmed cell death 1 receptor, CTLA-4, Tumor mutation burden, and Immune signature". We also examined the references of the selected articles.

\section{Results}

\subsection{Molecular Biology of TNBC}

TNBC is considered a heterogeneous neoplasm with regard to molecular aberrations. Analysis of genomic expression profile of TNBC has delineated 4 subtypes, including: Basal like 1 (BL1), Basal like 2 (BL2), Luminal androgen receptor (LAR), and Mesenchymal (M) (11,12). BL1 constitutes about 35\% of TNBCs and express cell cycle and DNA repair genes. BL2 constitutes $22 \%$ of TNBCs and express genes involved in the signal transduction of growth factors (epidermal growth factor (EGF), insulin-like growth factor 1 (IGF1), Wnt $\beta$-catenin), growth factor receptors (epidermal growth factor receptor (EGFR), MET, insulin-like growth factor 1 receptor (IGF1R), EPH receptor A2 (EPHA2)), glycolysis and gluconeogenesis pathways. LAR constitutes $16 \%$ of TNBC and expresses androgen receptor and luminal genes. $M$ constitutes $25 \%$ of TNBC and expresses genes involved in epithelial mesenchymal transition (EMT), cellular movement and differentiation, cancer stem cell regulation and growth factor signal transduction. This vast amount of heterogeneity in TNBC neoplasms necessitates personalization of treatment of TNBC based on novel discoveries in precision oncology.

About $10 \%$ to $20 \%$ of patients with TNBC carry germline mutations in BRCA1 gene. In addition, in patients who are negative for germline BRCA1 mutation, somatic mutations in homologous recombination pathway can create a similar phenotype named "BRCAness" (13). Increased response rate to genotoxic treatments such as platiniumbased agents like carboplatin and cisplatin, have been detected both in carriers of BRCA1 mutations and in patients with tumors showing BRCAness phenotype (14).

TNBC tumors show high genetic instability, with median mutation number of 1.7 in 1 million bases (range: 0.16 $5.23)(15,16)$. In addition, complex copy number alteration (CNAs) and structural rearrangement have been detected in TNBCs (17). There is a vast amount of variation in the genes mutated in TNBCs. Although some TNBCs have limited somatic mutations, in most TNBC neoplasms, a high rate of mutations have been detected in genes involved in signal transduction pathways (18). The most frequent mutated genes in TNBCs are TP53 and PIK3CA which are mutated in $82 \%$ and $10 \%$ of these tumors, respectively (19). However, in contrast to ER positive breast cancers, somatic mutations in TP53 in TNBCs are mostly nonsense single nucleotide variants and indels $(18,19)$. Somatic mutations in other known cancer driver genes, including PTEN, RB1,
$N F 1, B R C A 1, B R C A 2, E R B B 3, E R B B 4$, and $A L K$, have also been detected in TNBC neoplasms (17).

\subsection{Immune Signature in TNBC}

Pathologic evaluation of breast tumors have shown the diversity and clinical significance of leukocytic infiltration in different breast neoplasms $(20,21)$. Tumor infiltrating lymphocytes (TILs) are detected more in TNBCs, compared to other breast cancer types. It has been shown that the amount of CD8 positive T cells in TNBCs, when completely infiltrated within tumoral tissue, not just detected in stroma around the tumor, is an independent predictor of overall survival in multivariate analysis $(20,22,23)$. Infiltration of follicular CD4 positive helper T cells, which probably shows the existence of structured tertiary lymphoid structures (TLS) within tumoral tissue, is also a predictor of better overall survival in $\operatorname{TNBC}(24,25)$. Detection of regulatory T cells (Tregs) is considered a negative predictor of survival in TNBC (26).

Several studies have shown the association between increased expression of genes involved in immune response pathways and decreased risk of breast cancer recurrence (27-30). There is association between tumoral immune signature and metastasis risk in breast cancer. Tumors with increased expression of B cell/plasma cell, T cell and natural killer cell, monocyte and dendritic cell associated genes show lower metastasis risk, while tumors with decreased expression of any immune cell group show higher metastasis risk (31).

\subsection{Immunotherapy in TNBC}

The main concept of immunotherapy is to stimulate the immune system against the tumor in order to enhance its recognition and destruction by immune cells. Up to now, 2 immunotherapy strategies have been used in completed or ongoing clinical trials in TNBC, including immune checkpoint blockers and therapeutic cancer vaccines, which will be described briefly (Table 1).

\subsubsection{Immune Checkpoint Blockers}

Monoclonal antibodies against suppressive immunoregulatory mechanisms of response to tumorassociated antigens has been approved for various cancers, including melanoma, Non-small cell lung cancer, head and neck cancer, bladder cancer, lymphoma, and colorectal cancer (32). The most widely used targets are PD1, PDL1, and CTLA4.

During $\mathrm{T}$ cell activation, $\mathrm{T}$ cell receptor (TCR) binds with antigen presented by major histocompatibility antigen (MHC), but additional costimulatory signals are necessary. B7-1 (CD80) and B7-2 (CD86) on the antigen presenting cell (APC) bind with CD28 on the T cell, which leads to $T$ cell proliferation and differentiation through production of cytokines like interleukin 2 (IL2). CTLA4 is a homolog of 


\begin{tabular}{|c|c|c|c|}
\hline Agent & Clinical Trial Id & Phase & Recruitment Status \\
\hline \multicolumn{4}{|l|}{ Immune checkpoint blockers } \\
\hline Tremelimumab (CTLA4 antibody) & NCT02527434 & II & Active, not recruiting \\
\hline Combination of Durvalumab (PDL1 antibody) and Tremelimumab (CTLA4 antibody) & NCT02658214 & $\mathrm{Ib}$ & Recruiting \\
\hline Pembrolizumab (PD1 antibody) & NCT01848834 & $\mathrm{Ib}$ & Active, not recruiting \\
\hline Pembrolizumab (PD1 antibody) & NCT02447003 & II & Active, not recruiting \\
\hline Pembrolizumab (PD1 antibody) & NCT02555657 & III & Active, not recruiting \\
\hline Nivolumab (PD1 antibody) & NCT02393794 & $\mathrm{I} / \mathrm{II}$ & Recruiting \\
\hline Combination of Nivolumab (PD1 antibody) and Cabozantinib (Tyrosine kinase inhibitor) & NCT03316586 & II & Recruiting \\
\hline Atezolizumab (PDL1 antibody) & NCT03125902 & III & Recruiting \\
\hline Atezolizumab (PDL1 antibody) & NCT02425891 & III & Active, not recruiting \\
\hline \multicolumn{4}{|l|}{ Therapeutic cancer vaccines } \\
\hline Personalized peptide vaccine (PPV) & UMIN000001844 & II & Recruiting \\
\hline Dendritic cell (DC) vaccine & NCT02018458 & $\mathrm{I} / \mathrm{II}$ & Unknown \\
\hline MUC1 vaccine & NCT00986609 & I & Completed \\
\hline Folate receptor alpha peptide vaccine & NCT02593227 & II & Active, not recruiting \\
\hline
\end{tabular}

$\mathrm{CD} 28$ with increased affinity to $\mathrm{B} 7$ (33). The relative amount of CD28:B7 versus CTLA4:B7 is determinant of alternative paths of T cell activation or anergy (34). Antibodies against CTLA4 provoke antitumor immunity through phosphorylation of Akt and inhibition of Foxp3+ Treg cells (35).

Tremelimumab is a fully human monoclonal antibody specific for CTLA4. A phase II, open label, multicenter clinical trial (NCT02527434) is going to study Tremelimumab monotherapy in patients with advanced solid tumors including triple negative breast cancers. This study will analyze the safety and efficacy of Tremelimumab in the treatment of different patients with advanced cancer. If patients develop disease progression on Tremelimumab, they will receive Durvalumab (PDL1 inhibitor) or Tremelimumab and Durvalumab combination (36). A phase Ib study (NCT02658214) will determine the tolerability and safety of Durvalumab and Tremelimumab combined with first-line chemotherapy in patients with advanced solid tumors (37).

PD1, which is a member of B7 family, binds to its ligands (PDL1 and PDL2) and inhibits T cell proliferation, and IL2, interferon $\gamma$ (IFN $\gamma$ ) and tumor necrosis factor $\alpha$ (TNF $\alpha)$ production (38). Pembrolizumab is a humanized monoclonal antibody against PD1. KEYNOTE-012 trial (NCT01848834) investigated the safety and efficacy of pembrolizumab in advanced solid tumors (including a cohort of patients with triple negative breast cancer). This phase Ib trial studied 111 patients with TNBC, 58.6\% of whom had PDL1 positive tumors (defined as PDL1 expression in stroma or in $\geq 1 \%$ of tumor cells). In 27 patients who were selected for treatment and subsequently were analyzed for anti-tumor activity, the overall response rate was $18.5 \%$ (39). A phase II study is examining the efficacy and safety of pembrolizumab monotherapy in patients with metastatic TNBC (KEYNOTE086, NCT02447003) (40). KEYNOTE-119 (NCT02555657) is an ongoing phase III randomized trial which is analyzing patients with metastatic TNBC who has received single agent Pembrolizumab versus single agent chemotherapy (41).

Nivolumab is a fully human monoclonal antibody against PD1. A phase I/II non-randomized study is examining Cisplatin plus Romidepsin (histone deacetylase (HDCA) inhibitor) and Nivolumab in metastatic TNBC or $B R C A$ mutation-associated locally recurrent or metastatic breast cancer (NCT02393794) (42). Another phase II study is analyzing combination of Nivolumab with Cabozantinib (a non-specific tyrosine kinase inhibitor) in metastatic TNBC (NCT03316586) (43). Atezolizumab is a fully humanized monoclonal antibody specific for PDL1. An ongoing phase III multicenter randomized, double bind, placebo controlled trial is studying Atezolizumab in combination with paclitaxel compared to placebo with paclitaxel in patients with inoperable locally advanced or metastatic TNBC (NCT03125902) (44). Another phase III multicenter randomized, double bind, placebo controlled trial is examining Atezolizumab in combination with Nab-paclitaxel compared to placebo with Nab-paclitaxel in patients with metastatic TNBC (NCT02425891) (45).

\subsubsection{Therapeutic Cancer Vaccines}

To date, several therapeutic cancer vaccination strategies have been used to treat TNBC. A phase II trial examined personalized peptide vaccination (PPV) in metastatic 
TNBC. In this trial, vaccine antigens were selected from a pool of candidate peptides on the basis of pre-existing immunity. This PPV regimen boosted the immune response (both cytotoxic T lymphocyte (CTL) and IgG response) and resulted in possible clinical benefit (46). A phase I/II trial examined the safety of combination of preoperative chemotherapy with dendritic cell (DC) vaccination in patients with locally advanced TNBC(NCT02018458) (47). Preliminary results of this study showed that DC vaccination during preoperative chemotherapy is safe in TNBC patients (48).

A pilot study evaluated the efficacy of MUC1 peptide in boosting the immune response in stage I-III TNBC (NCT00986609). This early phase I study also analyzed the safety of MUC1 peptide-poly-ICLC adjuvant vaccine. (49). MUC1 is a member of the transmembrane mucin family, which are normally expressed on the gland-forming epithelial cells. Upon malignant transformation of these cells, hypoglycosylated mucins are produced which are recognizable by the immune system (50). The results of this study has not been published yet. A randomized multi-center phase II trial is currently examining the safety and immunogenicity of folate receptor alpha (FR $\alpha$ ) peptide vaccine mixed with a vaccine adjuvant (granulocytemacrophage colony stimulating factor (GM-CSF)) in stage IIb-III TNBC (NCT02593227) (51). It has been shown that $86 \%$ of TNBCs overexpress FR $\alpha$ (contrary to the limited expression in normal tissues) and its expression is associated with poor prognosis and increases the risk of recurrence $(52,53)$.

\subsection{Potential Biomarkers for Immunotherapy}

The ultimate goal of personalized cancer therapy is therapy selection based on individual patient characteristics. Given the high variability of breast cancers, it is important to find predictive biomarkers to select best treatment for the individual patient (54-57). Finding immunotherapy biomarkers will spare non-responding patients from ineffective expensive treatments and their adverse side effects. Tumor PDL1 expression is the most widely used immunotherapy biomarker. PDL1 is mostly expressed on dendritic cells and antigen presenting macrophages and binds to PD1 on activated T cells. Analysis of PDL1 expression by immunohistochemistry (IHC) is approved by the United States Food and Drug Administration (FDA) as companion diagnostic testing for pembrolizumab in several cancers $(58,59)$. However, multiple factors complicate the interpretation of PDL1 expression by IHC. Multiple PDL1 antibodies have been developed, but their comparative performance characteristics are not known. There is not a clear definition of "positive PDL1 staining", with cut-off points varying from $>1 \%$ to $50 \%$. In addition, there are limited antibody binding sites on PDL1, because it only has two hydrophilic regions. Therefore, its immunohistochemical detection in formalin-fixed paraffin embedded (FFPE) samples is not much effective (60). PDL1 expression as a biomarker of immunotherapy response is imperfect and it is necessary to find improved biomarkers $(61,62)$.

Neoplastic transformation is due to the accumulation of somatic mutations in tumor cells. There is considerable variation in the frequency of somatic alteration between individual tumors (63-65). It seems that tumor mutation burden (TMB) can be a promising biomarker of response to immunotherapy $(66,67)$. Some of the somatic mutations can produce neoantigens, which are recognized by the immune system and trigger an immune response that destroys neoplastic cells, especially after therapies that lead to $\mathrm{T}$ cell activation $(68,69)$. It is important to note that not all somatic mutations produce new peptides presented on the surface of major histocompatibility complex (MHC) molecules and not all neopeptides presented are immunogenic $(70,71)$. However, the more somatic mutations a cancer cell has, the more neoantigens it probably produces. Analysis of TMB can give an estimate of tumor mutation load.

Increased somatic mutation load has also been observed in tumors with impaired mismatch repair (MMR). MMR system controls the integrity of the genome. MMR proteins repair single base mismatches (insertions or deletions) which are produced during DNA replication, thus maintains the stability of the genome (72). It has been shown that MMR deficient tumors respond to immunotherapy irrespective of histologic type or tumor anatomic location (73). Therefore, MMR status can be a potential biomarker of response to immunotherapy.

\section{Conclusions}

TNBC is considered the subtype of breast cancer that is most likely to benefit from immunotherapy. As TNBC is regarded a heterogeneous disease, the discovery of biomarkers of response to immunotherapy will increase the likelihood of response to these therapies. Further in-depth investigations are needed to find novel biomarkers of response to these immunotherapies for the better management of patients with TNBC.

\section{Acknowledgments}

None declared.

\section{Footnotes}

Authors' Contribution: All authors designed the study. Sanaz Tabarestani reviewed the literature and wrote the manuscript. All authors approved the final manuscript.

Conflict of Interests: None declared.

Financial Disclosure: None declared. 


\section{References}

1. Bray F, Ferlay J, Soerjomataram I, Siegel RL, Torre LA, Jemal A. Global cancer statistics 2018: GLOBOCAN estimates of incidence and mortality worldwide for 36 cancers in 185 countries. CA Cancer J Clin. 2018;68(6):394-424. doi:10.3322/caac.21492. [PubMed: 30207593].

2. Bauer KR, Brown M, Cress RD, Parise CA, Caggiano V. Descriptive analysis of estrogen receptor (ER)-negative, progesterone receptor (PR)-negative, and HER2-negative invasive breast cancer, the so-called triple-negative phenotype: A population-based study from the California cancer Registry. Cancer. 2007;109(9):1721-8. doi: 10.1002/cncr.22618. [PubMed: 17387718].

3. Kohler BA, Sherman RL, Howlader N, Jemal A, Ryerson AB, Henry $\mathrm{KA}$, et al. Annual report to the nation on the status of cancer, 1975 - 2011, featuring incidence of breast cancer subtypes by race/ethnicity, poverty, and state.J Natl Cancer Inst. 2015;107(6):djv048. doi: 10.1093/jnci/djv048. [PubMed: 25825511]. [PubMed Central: PMC4603551].

4. Dent R, Trudeau M, Pritchard KI, Hanna WM, Kahn HK, Sawka CA, et al. Triple-negative breast cancer: Clinical features and patterns of recurrence. Clin Cancer Res. 2007;13(15 Pt 1):4429-34. doi: 10.1158/10780432.CCR-06-3045. [PubMed: 17671126].

5. Liedtke C, Mazouni C, Hess KR, Andre F, Tordai A, Mejia JA, et al. Response to neoadjuvant therapy and long-term survival in patients with triple-negative breast cancer.JClin Oncol.2008;26(8):1275-81. doi: 10.1200/JCO.2007.14.4147. [PubMed: 18250347].

6. Yousefi Kashi AS, Yazdanfar S, Akbari M, Rakhsha A. Triple negative breast cancer in iranian women: Clinical profile and survival study. Int J Cancer Manag. 2017;10(8). doi: 10.5812/ijcm.10471.

7. Sharma P. Biology and management of patients with triplenegative breast cancer. Oncologist. 2016;21(9):1050-62. doi: 10.1634/theoncologist.2016-0067. [PubMed: 27401886]. [PubMed Central: PMC5016071].

8. Denkert C, Liedtke C, Tutt A, von Minckwitz G. Molecular alterations in triple-negative breast cancer-the road to new treatment strategies. Lancet. 2017;389(10087):2430-42. doi: 10.1016/S0140-6736(16)32454-0.

9. Kwa MJ, Adams S. Checkpoint inhibitors in triple-negative breast cancer (TNBC): Where to go from here. Cancer. 2018;124(10):2086-103. doi: 10.1002/cncr.31272. [PubMed: 29424936]

10. Braun DA, Burke KP, Van Allen EM. Genomic approaches to understanding response and resistance to immunotherapy. Clin Cancer Res. 2016;22(23):5642-50. doi: 10.1158/1078-0432.CCR-16-0066. [PubMed: 27698000]. [PubMed Central: PMC5135569].

11. Lehmann BD, Bauer JA, Chen X, Sanders ME, Chakravarthy AB, Shyr $\mathrm{Y}$, et al. Identification of human triple-negative breast cancer subtypes and preclinical models for selection of targeted therapies.J Clin Invest. 2011;121(7):2750-67. doi: 10.1172/JCI45014. [PubMed: 21633166]. [PubMed Central: PMC3127435].

12. Lehmann BD, Jovanovic B, Chen X, Estrada MV, Johnson KN, Shyr $\mathrm{Y}$, et al. Refinement of triple-negative breast cancer molecular subtypes: Implications for neoadjuvant chemotherapy selection. PLoS One. 2016;11(6). e0157368. doi: 10.1371/journal.pone.0157368. [PubMed: 27310713]. [PubMed Central: PMC4911051].

13. Lord CJ, Ashworth A. BRCAness revisited. Nat Rev Cancer. 2016;16(2):110-20. doi: 10.1038/nrc.2015.21. [PubMed: 26775620].

14. Telli ML, Timms KM, Reid J, Hennessy B, Mills GB, Jensen KC, et al. Homologous recombination deficiency (HRD) score predicts response to platinum-containing neoadjuvant chemotherapy in patients with triple-negative breast cancer. Clin Cancer Res. 2016;22(15):3764-73. doi: 10.1158/1078-0432.CCR-15-2477. [PubMed: 26957554].

15. Kandoth C, McLellan MD, Vandin F, Ye K, Niu B, Lu C, et al. Mutational landscape and significance across 12 major cancer types. Nature. 2013;502(7471):333-9. doi: 10.1038/nature12634. [PubMed: 24132290]. [PubMed Central: PMC3927368].

16. Ng CKY, Schultheis AM, Bidard FC, Weigelt B, Reis-Filho JS. Breast cancer genomics from microarrays to massively parallel sequencing: Paradigms and new insights. J Natl Cancer Inst. 2015;107(5). doi: 10.1093/jnci/djv015.

17. Pareja F, Geyer FC, Marchio C, Burke KA, Weigelt B, Reis-Filho JS. Triplenegative breast cancer: The importance of molecular and histologic subtyping, and recognition of low-grade variants. NPJ Breast Cancer. 2016;2:16036. doi: 10.1038/npjbcancer.2016.36. [PubMed: 28721389]. [PubMed Central: PMC5515338].

18. Shah SP, Roth A, Goya R, Oloumi A, Ha G, Zhao Y, et al. The clonal and mutational evolution spectrum of primary triple-negative breast cancers. Nature. 2012;486(7403):395-9. doi: 10.1038/nature10933. [PubMed: 22495314]. [PubMed Central: PMC3863681].

19. Cancer Genome Atlas N. Comprehensive molecular portraits of human breast tumours. Nature. 2012;490(7418):61-70. doi: 10.1038/nature11412. [PubMed: 23000897]. [PubMed Central: PMC3465532].

20. Savas P, Salgado R, Denkert C, Sotiriou C, Darcy PK, Smyth MJ, et al. Clinical relevance of host immunity in breast cancer: From TILs to the clinic. Nat Rev Clin Oncol. 2016;13(4):228-41. doi: 10.1038/nrclinonc.2015.215. [PubMed: 26667975].

21. Stanton SE, Adams S, Disis ML. Variation in the incidence and magnitude of tumor-infiltrating lymphocytes in breast cancer subtypes: A systematic review. JAMA Oncol. 2016;2(10):1354-60. doi: 10.1001/jamaoncol.2016.1061. [PubMed: 27355489].

22. Loi S, Sirtaine N, Piette F, Salgado R, Viale G, Van Eenoo F, et al. Prognostic and predictive value of tumor-infiltrating lymphocytes in a phase III randomized adjuvant breast cancer trial in node-positive breast cancer comparing the addition of docetaxel to doxorubicin with doxorubicin-based chemotherapy: BIG 02-98. J Clin Oncol. 2013;31(7):860-7. doi: 10.1200/JCO.2011.41.0902. [PubMed: 23341518].

23. Liu S, Lachapelle J, Leung S, Gao D, Foulkes WD, Nielsen TO. CD8+ lymphocyte infiltration is an independent favorable prognostic indicator in basal-like breast cancer. Breast Cancer Res. 2012;14(2):R48. doi: 10.1186/bcr3148. [PubMed: 22420471]. [PubMed Central: PMC3446382].

24. Gu-Trantien C, Loi S, Garaud S, Equeter C, Libin M, de Wind A, et al. CD4(+) follicular helper T cell infiltration predicts breast cancer survival. J Clin Invest. 2013;123(7):2873-92. doi: 10.1172/JCI67428. [PubMed: 23778140]. [PubMed Central: PMC3696556].

25. Martinet L, Garrido I, Filleron T, Le Guellec S, Bellard E, Fournie JJ, et al. Human solid tumors contain high endothelial venules: Association with T- and B-lymphocyte infiltration and favorable prognosis in breast cancer. Cancer Res. 2011;71(17):5678-87. doi: 10.1158/00085472.CAN-11-0431. [PubMed: 21846823].

26. Stanton SE, Disis ML. Clinical significance of tumor-infiltrating lymphocytes in breast cancer. J Immunother Cancer. 2016;4:59. doi: 10.1186/s40425-016-0165-6. [PubMed: 27777769]. [PubMed Central: PMC5067916].

27. Alexe G, Dalgin GS, Scanfeld D, Tamayo P, Mesirov JP, DeLisi C, et al. High expression of lymphocyte-associated genes in node-negative HER2+ breast cancers correlates with lower recurrence rates. Cancer Res. 2007;67(22):10669-76. doi: 10.1158/0008-5472.CAN-07-0539. [PubMed: 18006808].

28. Broet P, Kuznetsov VA, Bergh J, Liu ET, Miller LD. Identifying gene expression changes in breast cancer that distinguish early and late relapse among uncured patients. Bioinformatics. 2006;22(12):1477-85. doi: 10.1093/bioinformatics/btl110. [PubMed: 16551658].

29. Rody A, Holtrich U, Pusztai L, Liedtke C, Gaetje R, Ruckhaeberle E, et al. T-cell metagene predicts a favorable prognosis in estrogen receptor-negative and HER2-positive breast cancers. Breast Cancer Res. 2009;11(2):R15. doi: 10.1186/bcr2234. [PubMed: 19272155]. [PubMed Central: PMC2688939].

30. Schmidt M, Bohm D, von Torne C, Steiner E, Puhl A, Pilch H, et al. The humoral immune system has a key prognostic impact in node-negative breast cancer. Cancer Res. 2008;68(13):5405-13. doi: 10.1158/0008-5472.CAN-07-5206. [PubMed: 18593943].

31. Nagalla S, Chou JW, Willingham MC, Ruiz J, Vaughn JP, Dubey P, et al. Interactions between immunity, proliferation and molecular subtype in breast cancer prognosis. Genome Biol. 2013;14(4):R34. doi: 10.1186/gb-2013-14-4-r34. [PubMed: 23618380]. [PubMed Central: PMC3798758].

32. National Cancer Institute. FDA approvals - cancer currents blog. United States: National Cancer Institute (NCI); 2018, [cited April 5, 2018]. Available from: https://www.cancer.gov/newsevents/cancer-currents-blog/fda-approvals?startMonth= 
$\&$ startyear $=\&$ endMonth $=\&$ end Year $=\&$ page $=1 \&$ RecordsPerPage $=$ $50 \&$ Offset $=0$.

33. Chambers CA, Kuhns MS, Egen JG, Allison JP. CTLA-4-mediated inhibition in regulation of $\mathrm{T}$ cell responses: Mechanisms and manipulation in tumor immunotherapy. Annu Rev Immunol. 2001;19:565-94. doi: 10.1146/annurev.immunol.19.1.565. [PubMed: 11244047].

34. Buchbinder EI, Desai A. CTLA-4 and PD-1 pathways: Similarities, differences, and implications of their inhibition. Am J Clin Oncol. 2016;39(1):98-106. doi: 10.1097/COC.0000000000000239. [PubMed: 26558876]. [PubMed Central: PMC4892769].

35. Liu L, Wang Y, Miao L, Liu Q, Musetti S, Li J, et al. Combination immunotherapy of MUC1 mRNA nano-vaccine and CTLA-4 blockade effectively inhibits growth of triple negative breast cancer. Mol Ther. 2018;26(1):45-55. doi: 10.1016/j.ymthe.2017.10.020. [PubMed: 29258739]. [PubMed Central: PMC5763160].

36. Clinical Trials. Study of tremelimumab in patients with advanced solid tumors. United States: U. S. National Library of Medicine; [cited September 17, 2018]. Available from: https://clinicaltrials.gov/ct2/ show/NCT02527434.

37. Clinical Trials. Durvalumab and tremelimumab in combination with firstline chemotherapy in advanced solid tumors. United States: U. S. National Library of Medicine; [cited November 7, 2018]. Available from: https://clinicaltrials.gov/ct2/show/NCT02658214.

38. Keir ME, Butte MJ, Freeman GJ, Sharpe AH. PD-1 and its ligands in tolerance and immunity. Annu Rev Immunol. 2008;26:677-704. doi: 10.1146/annurev.immunol.26.021607.090331. [PubMed: 18173375].

39. Nanda R, Chow LQ, Dees EC, Berger R, Gupta S, Geva R, et al. Pembrolizumab in patients with advanced triple-negative breast cancer: Phase Ib KEYNOTE-012 study. J Clin Oncol. 2016;34(21):2460-7. doi: 10.1200/JCO.2015.64.8931. [PubMed: 27138582].

40. Clinical Trials. Study of pembrolizumab (MK-3475) monotherapy for metastatic triple-negative breast cancer (MK-3475-086/KEYNOTE-086). United States: U. S. National Library of Medicine; [cited November 27, 2017]. Available from: https://www.clinicaltrials.gov/ct2/show/ NCT02447003?term $=$ NCT02447003\&rank $=1$.

41. Clinical Trials. Study of single agent pembrolizumab (MK-3475) versus single agent chemotherapy for metastatic triple negative breast cancer (MK3475-119/KEYNOTE-119). United States: U. S. National Library of Medicine; [cited September 25, 2017]. Available from: https://clinicaltrials.gov/ ct2/show/NCT02555657?term=NCT02555657\&rank=1.

42. Clinical Trials. Cisplatin plus romidepsin \& nivolumab in locally recurrent or metastatic triple negative breast cancer (TNBC). United States: U. S. National Library of Medicine; [cited October 11, 2018]. Available from: https://clinicaltrials.gov/ct2/show/NCT02393794?term= Nivolumab\&cond=Triple+Negative + Breast + Cancer\&rank $=1$.

43. Clinical Trials. A phase II study of nivolumab in combination with cabozantinib for metastatic triple-negative breast cancer. United States: U. S. National Library of Medicine; [cited August 28, 2018]. Available from: https://clinicaltrials.gov/ct2/show/NCT03316586?term= Nivolumab\&cond $=$ Triple + Negative + Breast + Cancer.

44. Clinical Trials. A study of atezolizumab and paclitaxel versus placebo and paclitaxel in participants with previously untreated locally advanced or metastatic triple negative breast cancer (TNBC) (IMpassion131). United States: U. S. National Library of Medicine; [cited November 28, 2018]. Available from: https://clinicaltrials.gov/ct2/show/NCT03125902? term $=$ NCT03125902\&rank $=1$.

45. Clinical Trials. A study of atezolizumab in combination with nabpaclitaxel compared with placebo with nab-paclitaxel for participants with previously untreated metastatic triple-negative breast cancer(IMpassion130). United States: U. S. National Library of Medicine; [cited December 10, 2018]. Available from: https://clinicaltrials.gov/ct2/show/ NCT02425891?term $=$ NCT02425891\&rank=1.

46. Takahashi R, Toh U, Iwakuma N, Takenaka M, Otsuka H, Furukawa $\mathrm{M}$, et al. Feasibility study of personalized peptide vaccination for metastatic recurrent triple-negative breast cancer patients. Breast Cancer Res. 2014;16(4):R70. doi: 10.1186/bcr3685. [PubMed: 24992895]. [PubMed Central: PMC4227005].

47. Clinical Trials. Safety study of chemotherapy combined with dendritic cell vaccine to treat breast cancer. United States: U. S. National Li- brary of Medicine; [cited January 14, 2016]. Available from: https:// clinicaltrials.gov/ct2/show/NCT02018458.

48. O'Shaughnessy J, Roberts LK, Smith JL, Levin MK, Timis R, Finholt JP, et al. Safety and initial clinical efficacy of a dendritic cell (DC) vaccine in locally advanced, triple-negative breast cancer (TNBC) patients (pts).JClin Oncol.2016;34(15_suppl):1086.doi: 10.1200/JCO.2016.34.15_suppl.1086.

49. Clinical Trials. MUC1 vaccine for triple-negative breast cancer. United States: U. S. National Library of Medicine; [cited July 23, 2018]. Available from: https://clinicaltrials.gov/ct2/show/NCT00986609.

50. Siroy A, Abdul-Karim FW, Miedler J, Fong N, Fu P, Gilmore H, et al. MUC1 is expressed at high frequency in early-stage basal-like triple-negative breast cancer. Hum Pathol. 2013;44(10):2159-66. doi 10.1016/j.humpath.2013.04.010. [PubMed: 23845471]. [PubMed Central: PMC4167755].

51. Clinical Trials. Folate receptor alpha peptide vaccine with GM-CSF in patients with triple negative breast cancer. United States: U. S. National Library of Medicine; [cited November 17, 2017]. Available from: https://clinicaltrials.gov/ct2/show/NCT02593227?term= NCT02593227\&rank=1.

52. Zhang Z, Wang J, Tacha DE, Li P, Bremer RE, Chen H, et al. Folate receptor alpha associated with triple-negative breast cancer and poor prognosis. Arch Pathol Lab Med. 2014;138(7):890-5. doi: 10.5858/arpa.20130309-OA. [PubMed: 24028341].

53. Ginter PS, McIntire PJ, Cui X, Irshaid L, Liu Y, Chen Z, et al. Folate receptor alpha expression is associated with increased risk of recurrence in triple-negative breast cancer. Clin Breast Cancer. 2017;17(7):544-9. doi: 10.1016/j.clbc.2017.03.007. [PubMed: 28410844].

54. Tabarestani S, Motallebi M, Akbari ME, Moghani MM, Shojaee L. Analysis of BRCA1/2 mutations and performance of manchester scoring system in high risk iranian breast cancer patients: A pilot study. Int JCancer Manag. 2017;10(12). doi: 10.5812/ijcm.60392.

55. Tabarestani S, Motallebi M, Akbari ME. Are estrogen receptor genomic aberrations predictive of hormone therapy response in breast cancer? Iran J Cancer Prev. 2016;9(4). e6565. doi: 10.17795/ijcp-6565. [PubMed: 27761212]. [PubMed Central: PMC5056018].

56. Tabarestani S, Ghaderian SM, Rezvani H, Mirfakhraie R, Ebrahimi A, Attarian $\mathrm{H}$, et al. Prognostic and predictive value of copy number alterations in invasive breast cancer as determined by multiplex ligationdependent probe amplification. Cell Oncol (Dordr). 2014;37(2):107-18 doi: 10.1007/s13402-013-0165-1. [PubMed: 24573687].

57. Tabarestani S, Ghaderian SM, Rezvani H, Mirfakhraie R. Expression profiling of breast cancer patients treated with tamoxifen: Prognostic or predictive significance. Med Oncol. 2014;31(4):896. doi: 10.1007/s12032-014-0896-5. [PubMed: 24563328].

58. Reck M, Rodriguez-Abreu D, Robinson AG, Hui R, Csoszi T, Fulop A, et al. Pembrolizumab versus chemotherapy for PD-L1-positive non-small-cell lung cancer. N Engl J Med. 2016;375(19):1823-33. doi: 10.1056/NEJMoa1606774. [PubMed: 27718847].

59. Herbst RS, Baas P, Kim DW, Felip E, Perez-Gracia JL, Han JY, et al. Pembrolizumab versus docetaxel for previously treated, PD-L1-positive, advanced non-small-cell lung cancer (KEYNOTE-010): A randomised controlled trial. Lancet. 2016;387(10027):1540-50. doi: 10.1016/S01406736(15)01281-7.

60. Patel SP, Kurzrock R. PD-L1 Expression as a predictive biomarker in cancer immunotherapy. Mol Cancer Ther. 2015;14(4):847-56. doi: 10.1158/1535-7163.MCT-14-0983. [PubMed: 25695955].

61. Koemans WJ, Chalabi M, van Sandick JW, van Dieren JM, Kodach LL. Beyond the PD-L1 horizon: In search for a good biomarker to predict success of immunotherapy in gastric and esophageal adenocarcinoma. Cancer Lett. 2018;442:279-86. doi: 10.1016/j.canlet.2018.11.001. [PubMed: 30419350].

62. Chan TA, Yarchoan M, Jaffee E, Swanton C, Quezada SA, Stenzinger A, et al. Development of tumor mutation burden as an immunotherapy biomarker: Utility for the oncology clinic. Ann Oncol. 2018. doi: 10.1093/annonc/mdy495.

63. Lawrence MS, Stojanov P, Polak P, Kryukov GV, Cibulskis K, Sivachenko $\mathrm{A}$, et al. Mutational heterogeneity in cancer and the search for new cancer-associated genes. Nature. 2013;499(7457):214-8. 
doi: 10.1038/nature12213. [PubMed: 23770567]. [PubMed Central: PMC3919509].

64. Chalmers ZR, Connelly CF, Fabrizio D, Gay L, Ali SM, Ennis R, et al. Analysis of 100,000 human cancer genomes reveals the landscape of tumor mutational burden. Genome Med.2017;9(1):34. doi:10.1186/s13073 017-0424-2. [PubMed: 28420421]. [PubMed Central: PMC5395719].

65. Zehir A, Benayed R, Shah RH, Syed A, Middha S, Kim HR, et al. Mutational landscape of metastatic cancer revealed from prospective clinical sequencing of 10,000 patients. Nat Med. 2017;23(6):70313. doi: 10.1038/nm.4333. [PubMed: 28481359]. [PubMed Central: PMC5461196].

66. Rizvi NA, Hellmann MD, Snyder A, Kvistborg P, Makarov V, Havel JJ, et al. Cancer immunology. Mutational landscape determines sensitivity to PD-1 blockade in non-small cell lung cancer. Science. 2015;348(6230):124-8. doi: 10.1126/science.aaa1348. [PubMed: 25765070]. [PubMed Central: PMC4993154].

67. Snyder A, Makarov V, Merghoub T, Yuan J, Zaretsky IM, Desrichard A, et al. Genetic basis for clinical response to CTLA-4 blockade in melanoma. $N$ Engl J Med. 2014;371(23):2189-99. doi: 10.1056/NEJMoa1406498. [PubMed: 25409260]. [PubMed Central: PMC4315319].

68. Riaz N, Morris L, Havel JJ, Makarov V, Desrichard A, Chan TA. The role of neoantigens in response to immune checkpoint blockade. Int Immunol. 2016;28(8):411-9. doi: 10.1093/intimm/dxw019. [PubMed: 27048318]. [PubMed Central: PMC4986233].

69. Tabarestani S, Akbari ME, Namaki S. Genomics role in cancer immunosurveillance: Impact on immunotherapy response. Int J Cancer Manag. 2018;11(11). doi: 10.5812/ijcm.85552.

70. Coulie PG, Van den Eynde BJ, van der Bruggen P, Boon T. Tumour antigens recognized by $\mathrm{T}$ lymphocytes: At the core of cancer immunotherapy. Nat Rev Cancer. 2014;14(2):135-46. doi: 10.1038/nrc3670. [PubMed: 24457417].

71. Snyder A, Chan TA. Immunogenic peptide discovery in cancer genomes. Curr Opin Genet Dev. 2015;30:7-16. doi: 10.1016/j.gde.2014.12.003. [PubMed: 25588790].

72. Modrich P. Mechanisms in eukaryotic mismatch repair. J Biol Chem. 2006;281(41):30305-9. doi: 10.1074/jbc.R600022200. [PubMed: 16905530]. [PubMed Central: PMC2234602].

73. Le DT, Durham JN, Smith KN, Wang H, Bartlett BR, Aulakh LK, et al. Mismatch repair deficiency predicts response of solid tumors to PD-1 blockade. Science. 2017;357(6349):409-13. doi:10.1126/science.aan6733. [PubMed: 28596308]. [PubMed Central: PMC5576142]. 